\title{
Happiness as alchemy: Positive mood leads to self-serving responses to social comparisons
}

\author{
Camille S. Johnson • Diederik A. Stapel
}

Published online: 19 April 2011

(c) The Author(s) 2011. This article is published with open access at Springerlink.com

\begin{abstract}
People in a positive mood process information in ways that reinforce and maintain this positive mood. The current studies examine how positive mood influences responses to social comparisons and demonstrates that people in a positive mood interpret ambiguous information about comparison others in self-benefitting ways. Specifically, four experiments demonstrate that compared to negative mood or neutral mood participants, participants in a positive mood engage in effortful re-interpretations of ambiguously similar comparison targets so that they may assimilate to upward comparison targets and contrast from downward comparison targets.
\end{abstract}

Keywords Social comparison · Positive mood Self-evaluations - Assimilation and contrast . Self-serving bias

\section{Introduction}

All seasons are beautiful for the person who carries happiness within.

-Horace Friess

The quote above suggests that positive mood is associated with a sunny or benevolent outlook on life that leads to additional happiness. Indeed, there is a growing psychological literature demonstrating that positive mood begets

C. S. Johnson ( $\)$

San Jose State University, One Washington Square, San Jose, CA 95192-0070, USA

e-mail: Camille.Johnson@sjsu.edu

D. A. Stapel

Tilburg University, Tilburg, The Netherlands positive mood. For example, the broaden and build model (Fredrickson 2001) has shown that happiness can create an upward spiral in which positive emotions broaden attention and cognition. Because of this broader perceptual approach, happy individuals are able to find positive meaning in events, which increases the level of positive emotions. Similarly, work on mood-congruency has shown that happy individuals are more attentive to positive information in the environment and therefore may be more likely to find information that reinforces positive moods (Tamir and Robinson 2007). More recently, researchers have shown that individuals in a positive mood are able to transform mood-threatening tasks into mood-benefiting tasks (Hirt et al. 2008). With the current research, we extend the idea that happy individuals are able to find positivity in their environment and argue that happy individuals are alchemists, of a sort, able to convert ambiguity into positivity, to convert lead into gold.

Specifically, we focus on the role that mood may play in person perception-the perception of comparison targets. We argue that when comparisons are detrimental to selfevaluations, the cognitive flexibility afforded by a positive mood allows people to reinterpret ambiguous information about social comparison targets in a self-serving manner. In addition, we argue that such self-serving interpretations are limited to situations in which perceivers have both the opportunity (the presence of ambiguous information) and the resources (cognitive resources) to engage in self-serving re-interpretations of information about targets.

Responding to social comparisons

In a typical day, most people encounter a bevy of peers and colleagues with whom they could compare themselves. Such social comparisons are one means by which 
individuals gain information about their status or standing, particularly in the absence of objective criteria (Festinger 1954). In particular, individuals may engage in upward comparisons with more successful others as a means of learning about their deficiencies and how to remedy them or they may engage in downward comparisons with less successful others as a means of feeling more positive about themselves (Wills 1981; Wood 1989). Responses to social comparisons are driven by person perception-the perceptions of comparison targets-and subtle differences in how the target is perceived can have a significant influence on those responses. Encountering another person is not enough to ensure that people will gain information about themselves from the encounter. Rather, individuals, or perceivers, must view the other person as an appropriate target for comparison (Festinger 1954). If a target is deemed inappropriate for comparison, a casual encounter is unlikely to have lasting effects, if any at all. Therefore, perceptions of comparison targets as similar or different and as holding particular traits (e.g. unsuccessful, intelligent, attractive) are important in determining if perceivers will respond to the comparison.

In addition to determining whether a target is appropriate for comparison, perceptions also determine how individuals will respond to a target. Responses to comparison targets fall into two broad categories: Self-judgments may be assimilated towards the target or contrasted away from the target. How one categorizes or defines a comparison target is an important determinant of whether assimilation or contrast with a target occurs (see e.g., Lockwood and Kunda 1997; Johnson and Stapel 2007). For example, individuals assimilate with targets who are viewed as attainable and contrast with targets who are viewed as unattainable (Lockwood and Kunda 1997). In this way, small shifts in perceptions of comparison targets may have important consequences for self-judgments and behaviors.

Traditionally, it has been assumed that perceptions of comparison targets are relatively static and irrevocable; once a perceiver categorizes a target in a particular way, this categorization does not change. According to this assumption, responses to comparisons depend upon perceptions of the target. For example, as shown in the rightmost portion of Fig. 1, if perceptions of the target lead individuals to contrast their self-views with the target, contrast with both upward and downward comparison targets is expected, regardless of the impact on self-evaluations. In this way, responses to comparison targets are often assumed to be symmetric (Mussweiler 2003; Wood 1989).

Imagine that a student has determined that a classmate represents a standard of performance against which she should compare herself, leading her to focus on differences in levels of performance between herself and the target. Her self-evaluations should typically show a contrast effect, such that a successful classmate lowers self-evaluations and an unsuccessful classmate raises self-evaluations. This is what we call a symmetric response: contrast with both upward and downward comparisons. Correspondingly, if our student has determined that a classmate is similar to the self in an important way (e.g., they scored similarly well on recent IQ tests) or in an unimportant but distinctive way (e.g., they are born on the exact same day), this typically leads to a focus on similarities in performance (see Mussweiler 2003; Stapel and Marx 2007). The focus on similarities should lead to an assimilation effect on selfevaluations, such that a more successful classmate leads to raised self-evaluations and a less successful classmate leads to lowered self-evaluations. This is also a symmetric response: assimilation with both upward and downward comparisons. ${ }^{1}$

Recent research, however, has shown that asymmetric responses may also occur (Schwinghammer et al. 2006; Stapel and Johnson 2007; Stapel and Koomen 2001a, b). For example, Stapel and Johnson (2007) found that following threats to positive self-evaluations, individuals responded to ambiguous targets in a self-serving and asymmetric manner: contrast with less successful targets and assimilation with more successful targets. In this study, psychology majors learned about a social science major from the same university. The relationship between the fields of psychology and social science is ambiguous and could be resolved in a number of ways. How participants resolved this ambiguity depended on whether or not they had just experienced a threat to self-evaluations. For those under threat, when it was advantageous to stress similarity between the two majors (i.e., when the target was inferior), the target was perceived as an appropriate comparison target and they contrasted their self-evaluations with the target ("I am better than she is."). Conversely, when it was advantageous to stress dissimilarity between the two majors (i.e., when the target was superior), participants perceived the target was inappropriate for comparison and their self-evaluations were not changed ("She is different, not relevant"). Thus, perceptions of comparison targets as similar depended upon which perception best fit the selfevaluation maintenance goals of the participants.

These earlier studies found asymmetric responses to social comparison in response to self-evaluation threat,

\footnotetext{
1 This is not to say that people merely accept negative influences on self-evaluations. In fact, people are adept at avoiding and minimizing negative effects. However, most research on self-evaluation maintenance has focused on how individuals shift perceptions following comparison to make the comparison less valid, not on how individuals can shift their categorization and identification of the comparison targets to change from assimilation to contrast.
} 


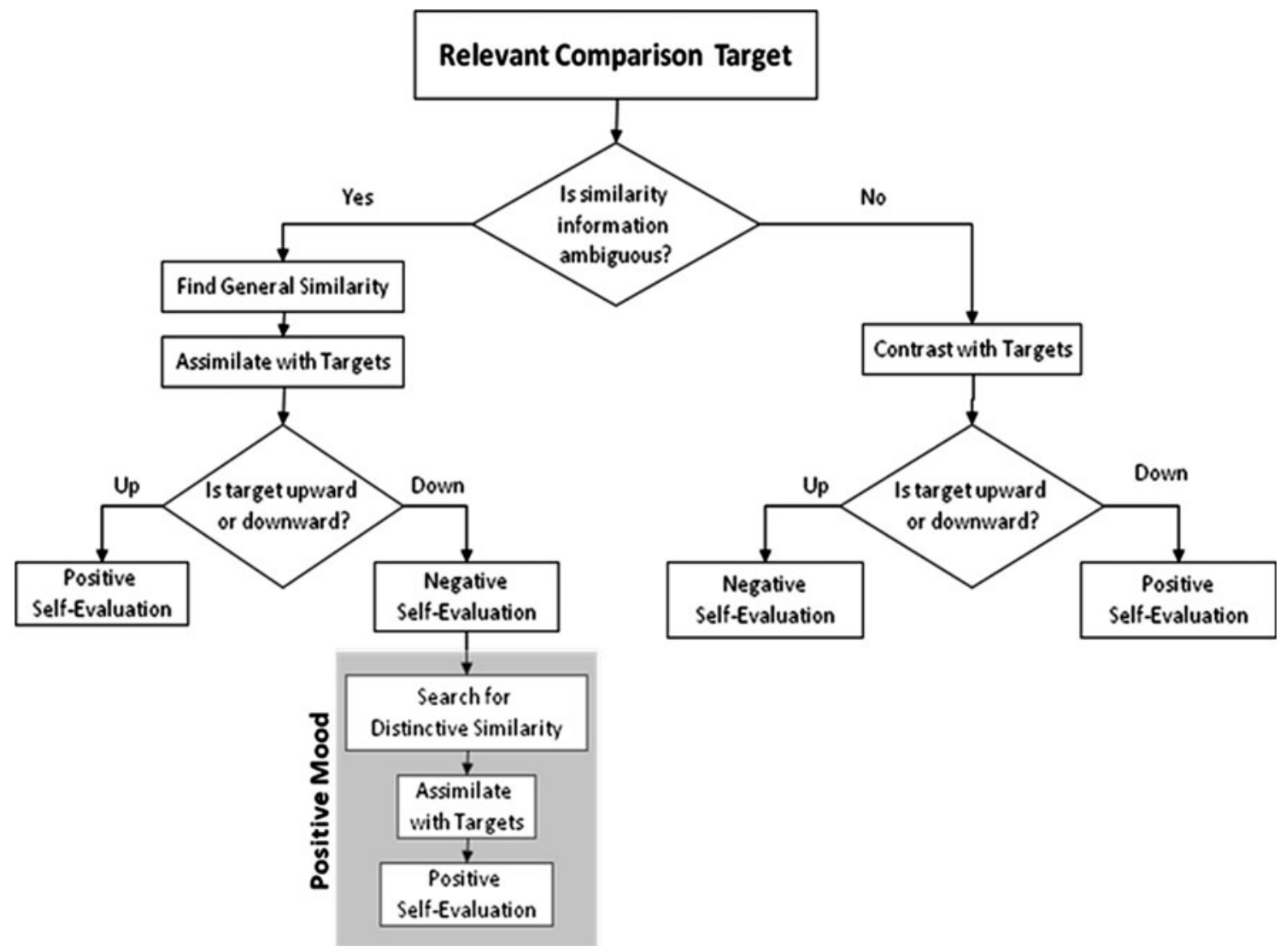

Fig. 1 Social comparison processes in neutral and negative mood (with the extra steps associated with positive mood inside the shaded box)

here, we suggest that positive mood may also lead to asymmetric responding. Our argument rests upon the rationale that just as positive mood states allow individuals to convert negative experiences into positive experiences through attention and identification of rewarding stimuli (Tamir and Robinson 2007); the flexible cognitive style associated with positive mood states may also facilitate the resolution of ambiguity in ways that maximize selfevaluations.

Positive mood, flexibility, and resolving ambiguity

Positive and negative moods are associated with different approaches to information processing (Clore et al. 2001; Fredrickson and Joiner 2002). Positive mood is associated with flexible, open, and global processing (Förster et al. 2008). For example, positive mood leads to increased flexibility (for reviews see Bless 2001; Martin and Clore 2001), creativity (Isen et al. 1987; Murray et al. 1990), and a preference for top-down as opposed to bottom-up processing of trait information (Isbell 2004). For instance, Murray et al. (1990) found that individuals in a positive mood were more cognitively flexible in categorizing objects. In that study, participants focused on similarities or differences between two television shows. Those in a positive mood listed more unique similarities and differences. That is, they were better able to make fine distinctions when comparing or contrasting two objects.

Given the relationship between threat and self-serving perceptions, one might expect that negative, and not positive mood, should be associated with self-serving perceptions. However, we argue that the cognitive abilities and tendencies associated with negative mood make them less likely. Negative mood may be characterized as vigilantly objective processing with rigorous attention to the environment and relative adherence to the data at hand (Schwarz 1990). Therefore, negative mood may impede the creative flexibility required for self-serving perceptions. For example, in a negative mood, individuals are less likely to abandon an established mental set (Gasper 2003), less likely to show false memory effects (Storbeck and Clore 2005), and are more attentive to local details (Avramova and Stapel 2008; Förster et al. 2008; Gasper and Clore 2002).

Building on this research, we suggest that the cognitive flexibility associated with positive mood may be used to categorize ambiguous comparison targets in self-serving ways. That is, when faced with a comparison that could 
harm self-views, individuals in a positive mood are better able to attend to minute details and draw fine distinctions that allow them to assimilate, contrast, or disregard the comparison information in order to benefit self-views.

\section{The steps leading to asymmetric responding}

The shaded area of Fig. 1 illustrates the steps to asymmetric responding. As suggested by earlier work (Stapel and Johnson 2007), asymmetric responses to social comparison occur when information about comparison targets is ambiguous. Whereas some information about comparison targets is concrete and not open to interpretation (e.g. biological sex), much of what perceivers know about others results from perceptual processes that can be guided by attitudes and preferences, such that even the perception of race can be altered (Caruso et al. 2009). Because of this, ambiguous information about targets (information that can be interpreted in more than one way) can be used strategically to benefit self-views. Specifically, we propose that in the presence of this ambiguous information, positive mood may initiate a process by which information about targets is reinterpreted until comparison with that target benefits self-evaluations.

\section{Current studies}

Our purpose is to understand how and when cognitive flexibility associated with positive mood allows individuals to interpret ambiguous information about comparison others in order to benefit self-evaluations. We briefly explore the consequences of negative mood, but focus our attention largely on illuminating the consequences of positive mood for social comparison. Fours studies examine how and when positive mood leads to self-serving responses to comparison targets.

Given that there are many ways in which perceptions of targets can be shifted in order to change the predominant response from contrast to assimilation (and vice versa) (Mussweiler 2003; Stapel and Koomen 2001a, b), the present studies capitalized on the differential effects of judging a target to be generally similar or distinctively similar (Stapel and Marx 2007). General similarity comes from sharing a common trait and leads perceivers to view a target as relevant enough to engage in contrastive comparisons ("you are similar enough for me compare with"). Alternatively, distinctive similarity is based on sharing an uncommon trait or having a unique or unusual connection (e.g. "we are both similarly different from others") that typically leads to assimilation. Imagine, for example, two college students. If both students dislike exams (a common trait among university students), this widespread background trait is likely to be used as the basis for further (contrastive) comparison processes on other trait dimensions. However, if both students play the tuba (a rare trait among university students), then they may be seen as distinctively and uniquely similar, and this unique similarity is likely to be generalized to other trait dimensions leading to assimilation.

As shown in the shaded portion of Fig. 1, individuals in a positive mood are expected to evaluate the distinctiveness of comparison targets in self-serving ways. Distinctive similarity information is particularly vulnerable to selfserving responding because information about distinctive similarity is relatively ambiguous and open to interpretation [people are inexperienced with using base rate information and judgments of prevalence are highly susceptible to irrelevant information (Kahneman and Tversky 1973)]. This ambiguity allows individuals to interpret distinctive similarity as meaningful when it concerns an upward standard (e.g., "we are uniquely similar and therefore share all kinds of traits"), but as meaningless when it concerns a downward standard (e.g., "what does playing the tuba have to do with math skills?"). By presenting participants with distinctive similarity information we can detect when selfserving interpretations are occurring.

In the present research, we first establish that manipulations of mood do not influence self-evaluations in the absence of social comparisons. In this pilot study, we tested a autobiographical mood induction procedure that has been employed by earlier research and shown to be effective (Krauth-Gruber and Ric 2000). Then, in Experiment 1, we tested the initial assumption of the model presented in Fig. 1: that ambiguous similarity information must be present in order for positive mood to lead to self-serving interpretations of that information. Specifically, in Experiment 1 , following a positive mood induction, participants viewed ambiguous similarity information or no information about a comparison target. We expected mood effects on responses to comparisons only when ambiguous similarity information was present. In the presence of ambiguous information, participants in a positive mood were expected to respond asymmetrically: assimilating to upward comparison targets and contrasting with downward targets. In the absence of ambiguous information, responses were expected to be symmetric, even if self-evaluations were harmed.

Experiment 2 replicates this effect and examines the effect of negative mood on responding. In Experiment 2, following a positive or negative mood induction manipulation, participants received ambiguous similarity or no information about a comparison target. Again, the responses of participants in a positive mood were expected to be asymmetric and self-serving: assimilation to upward comparison targets and contrast with downward targets. However, the responses of participants in a negative mood 
were expected to be symmetric: contrast with both upward and downward comparison others. In the absence of ambiguous information, responses in both positive and negative mood conditions were expected to be symmetric.

Next, we examined the boundaries of self-serving interpretations of ambiguous similarity information. In particular, we further tested our hypothesis that information must be ambiguous in order for self-serving responding to occur. Whereas in Experiment 1, we examined the effect of ambiguity by giving participants either ambiguous similarity information or no similarity information about a comparison standard, in Experiment 3 we examined the effect of ambiguity by giving participants similarity information that was either ambiguous or was explicitly non-ambiguous. Again, this corresponds to the first step in the model presented in Fig. 1. The inclusion of nonambiguous similarity information allowed us to test how flexible or creative people in a positive mood could be. Because the effects of positive mood are predicted to require ambiguous information, self-serving effects were expected to occur only when similarity information was ambiguous and open to alternative interpretation.

Finally, as shown in the shaded box in Fig. 1, reinterpretation of similarity information is hypothesized to occur only when initial interpretations yield negative self-evaluations, and is expected to be effortful. To test this, Experiment 4 examines how positive mood influences responses under conditions of reduced cognitive resources. Participants completed the mood induction task, were asked to hold in their memory an 8-digit number, and then viewed a comparison target. All participants were given ambiguous information about the target, and neutral mood control conditions were included. When participants were under load, the positive mood effects on responses to downward comparisons were expected to dissipate, reflecting the resource-demanding nature of self-serving asymmetric interpretations. Under load, responses of participants in a positive mood were expected to appear identical to those in a neutral mood.

\section{Pilot study}

The purpose of these studies is to demonstrate that positive mood influences the interpretations of comparison others in order to benefit self-evaluations. Thus, it was important to establish that mood, and the mood manipulations we employ, are not associated with changes in self-evaluations in the absence of comparison information. Therefore, a pilot study conducted in which participants $(N=45,30$ female, $M_{\text {age }}=19.5$ ) completed positive or negative mood manipulations in which they wrote brief essays describing an event that made them happy and still made them happy or describing an even that made them sad and still made them sad or a neutral filler task. This autobiographical method of manipulating mood has been found to be effective in creating long-lasting mood effects (Krauth-Gruber and Ric 2000; Mosak and Dreikurs 1973). Participants mood was measured using four nine-point items. Participants were asked to indicate, on bipolar scales, which word best described them (1) $1=$ negative mood, $9=$ positive mood; (2) $1=$ sad, $9=$ happy; (3) $1=$ unpleasant, 9 = pleasant; (4) $1=$ bad, $9=$ good (Wegener, Petty, and Smith, 1995). Then, they rated how well each of the following words described them on nine-point Likert scales ( $1=$ not at all me, $9=$ very much me): intelligent, competent, and successful. The four mood items were averaged to create an index of mood (Cronbach's alpha $=.91$ ) and the three self-ratings were averaged to create an index of self-evaluations (Cronbach's alpha $=.71$ ).

A one-way Analysis of Variance (ANOVA) revealed that the mood manipulation significantly affected participant mood, $F(2,42)=18.44, p<.001$. Participants in the positive mood condition reported more positive mood $(M=7.20, \mathrm{SD}=.93)$ that those in the control condition $(M=6.15, \mathrm{SD}=.52), t(42)=3.43, p=.001$. Participants in the negative mood condition reported more negative $\operatorname{mood}(M=4.98, \mathrm{SD}=.98)$ than those in the control condition, $t(42)=-3.80, p<.001$. No other effects were significant, $F \mathrm{~s}<1$. Thus, the manipulation of mood was effective.

A one-way ANOVA was also conducted on the index of self-evaluations. Those in the negative $\operatorname{mood}(M=6.98$, $\mathrm{SD}=.53)$, positive $\operatorname{mood}(M=6.98, \mathrm{SD}=.80)$, and the control condition $(M=6.82, \mathrm{SD}=.67)$, did not differ $F(1,42)=.27, p=.76$. Thus, manipulations of mood, alone, did not influence self-evaluations.

\section{Experiment 1}

To test the hypotheses that positive mood can influence responses to social comparison targets and that ambiguous similarity information must be present in order for selfserving responding to occur, participants in Experiment 1 completed a positive mood induction manipulation, received ambiguous or no similarity information about a comparison target, and completed self-evaluation measures. Ambiguous similarity information was expected to allow for asymmetric and self-serving responses: assimilation to upward comparison targets and contrast with downward targets. Absence of similarity information was expected to lead symmetric responding, even if self-evaluations were harmed.

Distinctive similarity was manipulating by presenting participants with targets with whom they did or did not share a birthday. A shared birthday is ambiguous similarity 
information. On the one hand, first hearing that you share a birthday with someone can lead to feelings of shared uniqueness, leading to assimilation, as has been shown in previous research (Brown et al. 1992; Cialdini and de Nicholas 1989; Finch and Cialdini 1989; see also Stapel and Marx 2007). On the other hand, sharing a birthday does not convey any information about shared experiences or other meaningful similarities, and may be irrelevant for determining similarity. Because the meaning of a shared birthday is ambiguous, we expect that the flexibility afforded by a positive mood will lead participants to treat upward comparison targets with whom they share a birthday as distinctively similar (leading to assimilation) and to treat downward comparison targets with whom they share a birthday as non-distinctively similar (leading to contrast).

Method

\section{Participants and design}

Female university $(N=47)$ students participated in the study for monetary compensation. Only female students were recruited to facilitate matching of target and participant gender. All participants received the positive mood manipulation and were distributed among a 2(direction of comparison: upward vs. downward) $\times 2$ (cue: shared birthday vs. different birthday) factorial design was used.

\section{Procedure}

Participants were recruited for what was described as several unrelated studies. Before beginning the experiment, participants completed a number of filler tasks in which they unscrambled words and named the capitals of European countries. Then, they completed the mood induction described in the pilot study.

After completing the mood manipulations, participants began the "impression-formation" task, during which they were exposed to the social comparison information. Via computer, participants were presented information about a target. They were told that at the end of the session, they would be interviewed about the efficacy of this method of person-information presentation. To manipulate direction of comparison, participants were first presented with a picture of an attractive or unattractive female student. The picture remained on screen while participants received information about the target, including physical characteristics, major, parents' names, and birth date. In the shared-birthday conditions, the presented information indicated that target had the same birthday and astrological sign as the participant. In the non-shared birthday conditions, a different birthday was given. This type of information has previously been used to establish general similarity and yield targets appropriate for comparison (Stapel and Marx 2007). After viewing this information, participants confirmed that they had processed all of the information, and were told that they would return to it later.

Dependent measures After completing the impression formation task, all participants reported their major, gender, and age. Participants also rated their own attractiveness (How attractive are you?) on a seven-point scale ( $1=$ not at all, $7=$ extremely). Then, participants rated the target's attractiveness on the same 7-point scale. Finally, participants were debriefed.

Results and discussion

Manipulation check

Confirming the efficacy of the direction of comparison manipulation, the upward comparison target was rated more attractive $(M=5.71, \mathrm{SD}=.75)$ than the downward target $(M=3.57, \mathrm{SD}=1.47), F(1,43)=38.80, p<.001$. No other effects on ratings of attractiveness were significant, $F \mathrm{~s}<1$.

\section{Self-evaluations}

Only when the ambiguous similarity cue (shared-birthday information) was present, were participants expected to respond strategically and asymmetrically: contrasting when it benefited self-evaluations (downward comparisons) and assimilating when it benefited self-evaluations (upward comparisons).

A 2(similarity cue: shared birthday vs. different birthday) $\times 2$ (direction: upward vs. downward) ANOVA revealed significant main effects of cue and direction of comparison such that those sharing a birthday reported higher self-evaluations $(M=4.92, \mathrm{SD}=1.14)$ than those not sharing a birthday $(M=3.96, \mathrm{SD}=1.3), F(1,43)=7.77$, $p=.008$. No other main effects were significant.

These main effects were qualified by the significant and expected interaction effect on self-evaluations, $F(1$, $43)=5.99, p=.019$. In the unshared birthday conditions, participants responded with symmetric contrast to the comparison targets. Exposure to the upward comparison target led to lowered self-evaluations $(M=3.33$, $\mathrm{SD}=.65)$ compared to the downward comparison target $(M=4.64, \mathrm{SD}=1.50), F(1,46)=6.95, p=011$.

However, in the shared birthday conditions, participant responses showed asymmetric assimilation towards the upward comparison target $(M=5.08, \mathrm{SD}=.52)$ and contrast away from the downward comparison target ( $M=4.75, \mathrm{SD}=1.54)$, such that direction of comparison did not significantly affect levels of self-evaluation, $F<1$. 
Experiment 1 provides a simple demonstration of selfserving responding when in a positive mood. Supporting our hypothesis, positive mood was associated with biased interpretations of ambiguous information (same birthday) that allowed for self-serving responses to both upward and downward comparison targets. However, in the absence of ambiguous information, positive mood was associated with symmetric responses to targets, leading to both positive and negative effects on self-views. The fact that ambiguous information was necessary for self-serving responding suggests that although positive mood is associated with flexibility, it is not associated with fabrication of reality. Thus, positive mood provides an interpretative lens, not a source of imagination.

\section{Experiment 2}

Having established that positive mood leads to asymmetric responding and that ambiguous information must be present for self-serving responding, Experiment 2 further explores the effect by including both negative mood and neutral mood conditions. Additional mood conditions are included for two reasons. First, we argue that positive mood leads to asymmetry in order to benefit self-views via a cognitive, information-processing route. However, one might argue that mood-congruent processing (Schwarz and Clore 1983; Tamir and Robinson 2007) is a more parsimonious explanation. The inclusion of negative mood allows us to test this alternative explanation. If asymmetric interpretations result from mood-congruent processing, negative mood should be associated with asymmetrically self-harming interpretations. However, if a flexible processing style is responsible for asymmetric responding in a positive mood, then the constrained and conservative processing associated with negative mood should lead to symmetric responses to comparisons. The inclusion of the negative mood condition also allows us to explore how the more conservative processing style associated with negative mood influences perceptions of ambiguous comparison targets.

Inclusion of a control condition in which participants neither completed the mood manipulation, nor viewed a comparison target, provides a baseline response that allows us to demonstrate absolute assimilation and contrast effects.

Method

\section{Participants and design}

University ( $N=141,75$ women) students participated in the study for monetary compensation. A 2(mood: positive vs. negative) $\times 2$ (direction of comparison: upward vs. downward $) \times 2$ (similarity cue: shared birthday vs. different birthday) factorial design was used, with an additional no-mood, no comparison control condition.

\section{Procedure}

The experimental procedures replicated those of Experiment 1 with the addition of negative mood and control conditions. As in Experiment 1, in the positive mood conditions, participants wrote about something that recently happened that made them very happy and still made them happy when they thought about it now. In the negative mood conditions, participants wrote about something that made them sad. In both conditions, they were asked to "go back to that time and think about how you felt then and describe your thoughts and feelings and what caused them. Try to hang on to the feeling to make it more intense." A control condition was included, in which participants completed only the filler tasks (e.g. naming European capitals) and self-ratings.

As in Experiment 1, after completing the mood manipulations, participants began the "impression-formation" task, in which they were exposed to the social comparison information. In the comparison conditions, in order to manipulate direction of comparisons, participants first saw a picture of an attractive or unattractive student (gender of the target was matched to the participant and different photos from those used in Experiment 1 were used) on computer. In the shared-birthday conditions, information about the targets indicated that the target had the same birthday and astrological sign as the participant. In the non-shared birthday conditions, a different birthday was indicated. After viewing this information, participants confirmed that they had processed all of the information, and were told that they would return to it later.

Dependent measures After completing the impression formation task, all participants reported their major, gender, age, and rated their own attractiveness and the target's attractiveness on seven-point scales $(1=$ not at all attractive and $7=$ extremely attractive). Participants also reported how positive or negative they felt at that moment ( $1=$ negative mood and $7=$ positive mood) (Stapel and Blanton 2004; Stapel and Koomen 2000). Finally, participants were debriefed.

In the no-comparison, no-mood induction control condition, participants completed only filler tasks, the selfevaluation measures, and the mood measures.

\section{Results and discussion}

Initial analyses examining the effect of sex of participant on mood and self-evaluations revealed no significant main 
effects or interactions. Therefore, we collapsed across participant sex in all reported analyses.

A no-mood induction, no-comparison hanging control condition was included to determine the direction of the effects on self-evaluations and mood. Therefore, analyses of self-evaluation and mood measures were conducted in two steps. First, a 2(mood: positive vs. negative) $\times$ 2(direction of comparison: upward vs. downward) $\times$ 2(cue: shared birthday vs. different birthday) ANOVA was conducted, testing for main effects and interactions. Then, following the strategy employed in previous research (Jaccard 1998), we conducted single degree of freedom contrasts comparing means in the factorial to the neutralmood, no-comparison condition to test if the observed effects were consistent with our specific hypotheses. ${ }^{2}$

\section{Manipulation check}

Confirming the efficacy of the mood manipulation, only the mood manipulation significantly affected mood, $F(1$, $132)=340.44, p<.001$. Participants in the positive mood condition were more positive $(M=5.72, \mathrm{SD}=.82)$ than the control condition $(M=4.40, \quad \mathrm{SD}=.83)$, $t(138)=5.61, p<.001$. The negative mood condition $(M=2.98, \mathrm{SD}=.83)$, was more negative than the control condition, $t(138)=-5.97, p<.001$.

The manipulation of direction of comparison was also effective. The upward comparison target was rated more attractive $(M=5.98, \mathrm{SD}=.69)$ than the downward target $(M=2.46, \mathrm{SD}=.74), F(1,118)=716.92, p<.001$. No other effects on ratings of attractiveness were significant, $F$ s $<1$.

\section{Self-evaluations}

The data from the experimental conditions were submitted to a 2(mood: positive vs. negative) $\times 2$ (direction: upward vs. downward) $\times 2$ (similarity cue: shared birthday vs. different birthday) ANOVA, which revealed the expected three-way interaction effect on self-evaluations, $F(1$, $118)=5.13, p=.03$. The analysis also revealed significant effects of similarity cue, $F(1,118)=21.84, p<.001$; direction, $F(1,118)=131.87, p<.001$; and mood, $F(1$,

\footnotetext{
2 These comparisons were conducted to test a priori hypotheses, and only those means relevant to the hypotheses were compared. However, in consideration of concerns about experiment wise alpha error, more conservative tests of all mean differences using the Tukey's HSD were conducted. These analyses revealed a similar pattern of significant differences from the control condition, with the only difference being that in the negative mood, shared birthday, upward comparison condition differs from the control at the $p=.07$ level as opposed to the $p<.05$ level.
}

$118)=14.48, p<.001$. In addition, the two-way interaction effects of mood and similarity cue, $F(1,118)=18.84$, $p<.001 ; \operatorname{mood}$ and direction, $F(1,118)=11.74, p=$ .001 ; and similarity cue and direction, $F(1,118)=14.11$, $p<.001$, were also significant. In order to interpret the three-way interaction, the positive and negative mood conditions are discussed separately and comparisons with the control condition are reported. All means and standard deviations are presented in Table 1.

Positive mood As can be seen in Table 1, in the positive mood conditions, direction of comparison, $F(1$, $124)=14.79, p<.001 ;$ presence of similarity cue (shared birthday or not), $F(1,124)=18.61, p<.001$; and the interaction effect of direction of comparison and similarity information, $F(1,124)=9.39, p=.003$, all significantly influenced self-ratings. As in Experiment 1, when participants believed they shared a birthday with the target, self-evaluations did not differ as a result of direction of comparison. Compared to the control condition, participants in both the upward, $F(1,132)=13.76, p<.001$, and downward, $F(1,132)=21.53, p<.001$, comparison conditions rated themselves more positively. That is, they asymmetrically assimilated to the upward comparison target and contrasted with the downward comparison target.

When participants believed that they did not share a birthday with the target, they responded symmetrically and contrasted with the targets, as in Experiment 1. Compared to the control condition, they lowered self-ratings after upward comparison, $F(1,132)=14.06, p<.001$ and raised self-ratings after downward comparison, $F(1,132)=$ $10.69, p=.001$.

Negative mood Among the negative mood conditions, only the main effect of direction was significant, $F(1$, $124)=56.97, p<.001$. That is, shared birthday information had no effect. All participants in a negative mood contrasted with the targets, resulting in lower ratings of the self after upward comparison and higher ratings of the self after downward comparison, relative to the control condition.

Supporting our hypothesis, positive mood was associated with biased interpretations of ambiguous information (same birthday) that allowed for self-serving responses to both upward and downward comparison targets. In contrast, negative mood was associated with unbiased and objective interpretations of ambiguous information. Across comparison conditions, participants in a negative mood treated shared-birthday information as irrelevant to the comparison and always contrasted with the targets. This pattern has two implications. First, it suggests that selfbeneficial responses to comparisons are not purely reparative processes; if they were, then negative mood might 
Table 1 Effects of upward (more attractive) versus downward (less attractive) comparisons on self-evaluations, ratings of target similarity, and use of information to benefit the self as a function of mood and shared birthday information

\begin{tabular}{|c|c|c|c|c|c|c|c|c|}
\hline & \multicolumn{4}{|c|}{ Positive mood } & \multicolumn{4}{|c|}{ Negative mood } \\
\hline & \multicolumn{2}{|c|}{ Same birthday } & \multicolumn{2}{|c|}{ Different birthday } & \multicolumn{2}{|c|}{ Same birthday } & \multicolumn{2}{|c|}{ Different birthday } \\
\hline & Upward & Downward & Upward & Downward & Upward & Downward & Upward & Downward \\
\hline Self-evaluation & $5.25(.93)$ & $5.53(.52)$ & $3.17(.98)$ & $5.13(.72)$ & $3.33(.90)$ & $5.20(.68)$ & $3.07(.62)$ & $5.35(.70)$ \\
\hline
\end{tabular}

From Experiment 2

Mean self-evaluations in the control condition were 4.20 with a standard deviation of .86 . All self-evaluations differed from the control condition at $p<.05$

have elicited them. ${ }^{3}$ Second, it suggests that asymmetrical responding does not result from mood-congruent processing. If mood-congruent processing were the underlying mechanism, then asymmetrically negative responses under negative mood (contrast with upward comparison targets and assimilation to downward) should have occurred.

In Experiments 1 and 2, in the non-shared birthday conditions, participants were provided with other information about the targets that could have been used in self-serving ways, but was not. We argue that this is because self-serving responding is limited to situations in which ambiguous information in present. Experiment 3 tests this hypothesis.

\section{Experiment 3}

Experiments 1 and 2 demonstrated that mood can influence the impact of social comparison information and that when in a positive mood, individuals interpret similarity cues in self-serving ways. In Experiments 1 and 2, participants either received ambiguous information or received no information about similarity at all (they had no reason to expect that they would share a birthday with the targets, thus not sharing a birthday might be considered the default belief and therefore not indicative of a lack of similarity or relevance). These experiments demonstrated that in the presence of ambiguous information, positive mood leads to self-serving responding. This does not prove that ambiguous information is necessary and this does not inform as to the degree to which positive mood tints their perceptions of the stimuli they encounter. In Experiment 3, in order to examine the limits of this interpretative bias, participants were presented with information that was explicitly described as ambiguous with regards to distinctive similarity or that was explicitly described as unambiguous with regards to distinctive similarity. Because we argue that self-beneficial responses to comparisons may only occur

\footnotetext{
3 This also suggests that negative mood is not harming selfevaluations, as previous research has shown that threats to selfevaluations lead to self-serving interpretations of ambiguous information.
}

when comparison targets are ambiguously similar and when fine distinctions such as those between distinctive and general similarity may be drawn, asymmetry in responding was only expected when the similarity cue was described as ambiguous.

In Experiment 3, the ambiguous information provided to participants was that they shared an artistic preference with a fellow participant (Stapel and Marx 2007). In the unambiguous conditions, sharing an artistic preference was described as being diagnostic of distinctive similarity. In these conditions, individuals in a positive mood were expected to act like those in the control conditions: symmetric assimilation effects were expected. In the ambiguous conditions, sharing an artistic preference was described as potentially diagnostic of distinctive similarity. Given this ambiguity, individuals in a positive mood were expected to act in self-serving ways. When faced with an upward comparison target, ambiguous information was expected to be viewed as indicating distinctive similarity, leading to assimilation and when faced with a downward comparison target, the ambiguous information was expected to be interpreted as providing no meaningful evidence of similarity, making the target irrelevant and leading to no response to the comparison target.

\section{Method}

\section{Participants}

University students $(N=96)$ participated in the study for course credit. Only women were recruited for the study to facilitate matching of participant and comparison target gender. A 2(mood: positive, neutral) $\times 2$ (direction of comparison: upward $\times$ downward) $\times 2$ (similarity cue: ambiguous vs. non-ambiguous) factorial design was used.

\section{Procedure}

Upon beginning the experiment, participants completed the same positive mood induction and neutral filler tasks as in Experiment 1. After completing the mood manipulations, 
participants completed a task called "Aesthetic Preferences." In this task, participants were shown four "artistic" line drawings and were told, "The following pictures have been rated by a large number of students. Please look at the following four pictures for a moment and circle the number of the picture that you feel is the most beautiful."

Prior to indicating their preference, participants in the ambiguous similarity cue condition, were told, "Some previous research has shown that aesthetic preferences reveal a great deal about people's unique personalities, while other research has shown that aesthetic preferences are useless in predicting anything about people's personalities. Science has yet to determine whether aesthetic preference reveal unique aspects of the person, provide general information, or say nothing about personalities. The question has yet to be settled." In the non-ambiguous cue condition, participants were told, "A great deal of previous research has shown that aesthetic preferences reveal a great deal about people's unique personalities and we are interested in expanding this line of research."

After participants indicated their preference, they began the same impression formation task as in Experiment 1 , in which they were exposed to the gender-matched comparison targets (and a different photo from that used in Experiments 1 and 2 was used for female participants). No birthday information was included. Instead, in both conditions, target information indicated that the target had chosen the same drawing in the aesthetic preference task.

Finally, participants answered some questions about themselves, ostensibly to determine whether their personality and mood had any impact on the tasks they had just completed. Participants reported their major, gender, age, and then rated their own attractiveness (on the seam measures used in Experiments 1 and 2). Then, participants rated the target on her attractiveness.

Participants also answered the following questions regarding similarity to the targets (Miller et al. 1988; Stapel and Marx 2007): (1) How similar are you and this person in general? $(1=$ not at all, $9=$ extremely similar $)$; (2) To what degree do you feel that there is a special bond between you and the other person? $(1=$ not at all, $9=$ very much); (3) How similar are you and this person in aesthetic preferences? $(1=$ not at all, $9=$ extremely similar).

\section{Results}

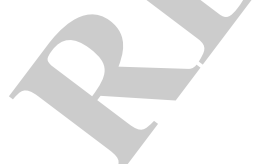

To test our hypotheses, a series of 2(ambiguity of cue: ambiguous vs. non-ambiguous) $\times 2$ (direction of comparison: up vs. down $) \times 2$ (mood: positive vs. control) ANOVAs were conducted on the dependent variables.

\section{Manipulation check}

Confirming the efficacy of the mood manipulation, only the main effect of mood condition significantly influenced selfreported mood, $F(1,88)=104.58, p<.001$. Participants in the positive mood condition were more positive $(M=$ 7.33, $\mathrm{SD}=.86)$ than the neutral condition $(M=5.44$, $\mathrm{SD}=.97)$.

The manipulation of direction of comparison was also effective. Only a main effect of direction of comparison emerged, such that the upward comparison target was rated more attractive $(M=7.33, \mathrm{SD}=1.19)$ than the downward target $\quad(M=4.17, \quad \mathrm{SD}=1.19), \quad F(1,88)=170.34$, $p<.001$.

\section{Self-evaluations}

In the unambiguous conditions (i.e. "a great deal of research has shown...") all participants were expected assimilate to the comparison targets and only the simple main effect of direction of comparison was expected to be significant.

In the ambiguous conditions (i.e. "science has yet to determine...") participants in a positive mood were expected to interpret the ambiguous information in selfserving ways, leading to asymmetric assimilation to upward comparison targets and contrast with downward comparison targets. Participants in the neutral mood condition were expected to interpret the ambiguous information as uniformly irrelevant, resulting in no response to the comparison targets.

In addition to these main effects, two two-way interaction effects were expected: mood $\times$ ambiguity and direction $\times$ ambiguity, and by specific comparison tests. Corresponding to the former interaction effect, when the participants were in a positive mood, they were expected to be uniformly more positive, regardless of direction of comparison, than when in a neutral mood. Corresponding to the latter interaction effect, When the information was not ambiguous, positive and neutral mood responses were expected to be the same. Therefore, mood was not expected to moderate this effect. Because of this, the three-way interactions were not expected to be significant.

To test these hypotheses, a 2(mood: positive vs. neutral) $\times 2$ (direction: upward vs. downward) $\times 2$ (similarity cue: unambiguous vs. ambiguous sign of distinctive similarity) ANOVA revealed significant effects of similarity cue $(F(1,88)=11.40, p=.001)$, direction $(F(1,88)=67.88$, $p<.001)$, and $\operatorname{mood}(F(1,88)=9.65, p=.003)$. These were qualified by the two-way interaction effects of similarity cue and $\operatorname{mood}(F(1,88)=19.87, p<.001)$ and similarity cue and direction $(F(1,88)=59.27, p<.001)$. Means for all conditions are presented in Table 2. 
Table 2 Effects of upward versus downward comparisons on self-evaluations and ratings of target similarity as function of mood and ambiguity of similarity information

\begin{tabular}{|c|c|c|c|c|c|c|c|c|}
\hline & \multicolumn{4}{|l|}{ Positive } & \multicolumn{4}{|l|}{ Neutral } \\
\hline & \multicolumn{2}{|l|}{ Ambiguous } & \multicolumn{2}{|c|}{ Unambiguous } & \multicolumn{2}{|l|}{ Ambiguous } & \multicolumn{2}{|c|}{ Unambiguous } \\
\hline & Upward & Downward & Upward & Downward & Upward & Downward & Upward & Downward \\
\hline Self-evaluation & $6.58(.90)$ & $6.50(.80)$ & $6.58(.52)$ & $4.08(.52)$ & $5.42(.79)$ & $5.33(.78)$ & $6.75(.87)$ & $4.33(.78)$ \\
\hline Similarity & $7.06(.53)$ & $4.14(.59)$ & $6.81(1.16)$ & $6.81(.63)$ & $5.72(.97)$ & $5.44(.69)$ & $6.72(.76)$ & $6.83(.80)$ \\
\hline
\end{tabular}

From Experiment 3

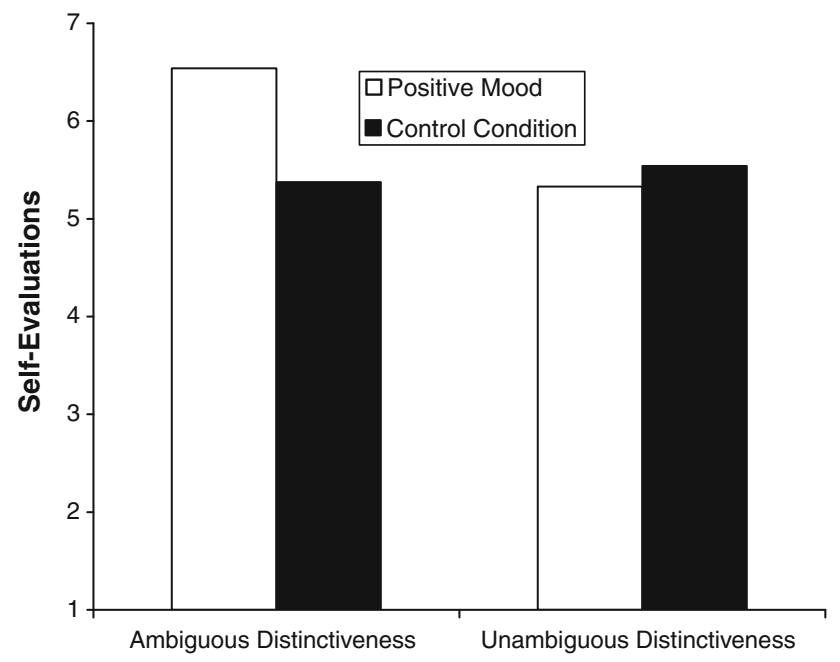

Fig. 2 In the presence of ambiguous distinctiveness information, positive mood leads to more positive self-evaluations, across upward and downward comparisons, than does neutral mood (from Experiment 3)

Similarity cue and mood effects on responses As seen in Fig. 2, our hypotheses were supported by the significant interaction effect of similarity cue and mood. When the similarity cue was unambiguous, responses of participants in the positive mood conditions did not respond in a selfserving manner and did not differ significantly from participants in the neutral mood conditions, regardless of the direction of comparison, $p>.05$.

When the similarity cue was ambiguous, participants in the positive mood reported significantly higher self-evaluations than those in the neutral mood condition, regardless of the direction of comparison, $F(1,94)=11.82, p=.001$, suggesting that they assimilated to the upward comparison targets, and contrasted from the downward comparison targets, resulting in uniformly more positive self-evaluations. That is, they regarded the aesthetic preference information as indicative of shared distinctiveness when the comparison was upward and as irrelevant when the comparison was downward. On the other hand, participants in a neutral mood interpreted the preference information as not indicative of distinctiveness or similarity and did not respond to the targets.
Similarity cue and direction of comparison effects on responses Examination of the interactive effect of similarity cue and direction reveals that when the similarity cue was ambiguous, no differences between upward and downward comparison conditions were found, $p>.05$. However, when the cue was unambiguous, participants assimilated to the comparison targets, as expected, $F(1$, 94) $=92.47, p<.001$.

\section{Perceptions of similarity}

In the presence of ambiguous similarity information, participants in a positive mood were expected to perceive themselves as more similar to upward comparison targets relative to downward comparison targets. To test these hypotheses, participant responses to the three similarity questions were averaged (alpha $=.87)$ and this similarity index was submitted to a 2(mood: positive vs. neutral) $\times 2$ (direction: upward vs. downward) $\times 2$ (similarity cue: unambiguous vs. ambiguous sign of distinctive similarity) ANOVA.

As shown in Table 2, the significant main effects of similarity cue, $F(1,88)=55.38, p<.001$ and direction, $F(1,88)=22.80, p<.001$, were qualified by the two-way interaction effects of direction and mood, $F(1,88)=18.14$, $p<.001$, and similarity cue and direction, $F(1,88)=$ $26.20, p<.001$. These were further qualified by the significant three-way interaction effect, $F(1,88)=15.32$, $p<.001$.

As shown in Table 2, and as revealed by planned comparisons, in the presence of ambiguous information, participants in a positive mood rated upward comparison targets as more similar to the self than downward comparison targets, $F(1,94)=53.17, p<.001$. However, when similarity information was unambiguous, participants rated the upward and downward comparison targets as equally similar to the self, $F(1,94)=1.00, p>.05$. Therefore, participants in a positive mood did not respond in a biased manner. In contrast, in the neutral mood condition, when information was ambiguous, direction of comparison did not influence ratings of similarity, $F(1,94)=.31, p=.58$. Identical effects 
emerged when the information was unambiguous, $F(1$, 94) $=1.50, p=.83$.

We further analyzed the relations between similarity and self-evaluations in each of the conditions. When information was unambiguous, self and other attractiveness were significantly related in the positive, $r(24)=.87, p<.001$, and neutral mood, $r(24)=.53, p=.008$, reflecting assimilation of self. In the ambiguous conditions, selfevaluations were not correlated with ratings of target attractiveness in either the positive or neutral mood, $p s>$.84. However, in a positive mood, ratings of similarity were correlated with ratings of other attractiveness, $r(24)=.70, p<.001$. In a neutral mood, this correlation was not significant, $p>.9$. These results suggest that the presence of ambiguous similarity cues allows those in a positive mood to see others in self-serving ways. ${ }^{4}$

\section{Discussion}

Supporting our hypothesis, positive mood was associated with biased interpretations of ambiguous information. When comparison targets were superior, sharing an esthetic preference was regarded as reflecting greater similarity, similarity was related to self-evaluations, and participants assimilated their self-evaluations to the targets. When comparison targets were inferior, sharing an esthetic preference was regarded as not reflecting similarity and participants did not respond to the targets. In this way, the resolution of ambiguity in a positive mood depended on how interpretations might affect self-evaluations. However, when the information was unambiguous, no differences in perceptions of sharing an esthetic preference were found. Whether the comparison target was superior or inferior, in both a positive and neutral mood, sharing a preference was regarded as highly reflective of similarity and participants assimilated to the targets.

\section{Experiment 4}

Experiment 3 demonstrated that positive mood only leads to self-serving responding when the distinctive similarity information is ambiguous. Experiment 4 extends these finding by testing the hypothesis that asymmetric responding in a positive mood results from effortful reinterpretation of ambiguous information, after initial processing leads to detrimental results. To test this hypothesis, participants completed a mood induction exercise, were asked to complete a cognitively effortful task, and then viewed a comparison target with whom they shared a birthday.

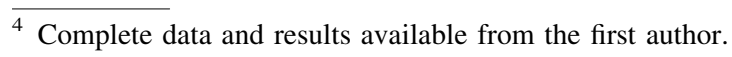

As in Experiments 1 and 2, participants were provided with ambiguous similarity information. Reinterpretation of this information was only expected when such reinterpretation was necessary and when individuals had sufficient cognitive resources. Consequently, for participants not under cognitive load, replication of the positive mood conditions from Experiments 1 and 2 was expected. However, for participants under load, replication of the neutral mood conditions from Experiment 2 was expected; lack of cognitive resources was expected to disrupt reinterpretation of ambiguous similarity information, thus preventing participants from responding positively to the downward comparison condition.

\section{Method}

\section{Participants and design}

University ( $N=193,103$ female) students participated in the study for monetary compensation. This experiment used a 2(mood: positive, neutral) $\times 2$ (direction of comparison: upward vs. downward) $\times 2$ (load: load vs. no load) factorial design, with an additional no mood induction, no comparison, no load control condition.

\section{Procedure}

The experimental procedures were the same as in Experiments 1 and 2. As in Experiment 1, participants completed either the happy or neutral mood exercise; there was no negative mood condition. Second, all participants viewed information about an attractive or unattractive comparison target which included information that they shared a birthday with the comparison target. Third, participants in the cognitive load conditions were asked to remember an 8-digit number and to be prepared to report the number when the experiment was finished (see Gilbert, Giesler, and Morris 1995). Participants were not allowed to write down or otherwise record that number, but were asked to hold it in mind throughout the rest of the study. All participants were able to recall the number at the end of the session. Participants in the no load conditions were not given a number to rehearse.

After viewing the comparison targets, participants then completed the same measures of mood, own attractiveness, and other attractiveness as in Experiments 1 and 2.

Results and discussion

As in Experiment 2, a hanging control (no load, no mood, no comparison) condition was included in this study and the same analysis strategy was employed. First, a 2(load: 
load vs. no load) $\times 2$ (mood: positive, neutral $) \times 2$ (direction of comparison: upward vs. downward) ANOVA was conducted, testing for main effects and interactions, followed by single degree of freedom contrasts to test specific hypotheses (Jaccard 1998).

\section{Manipulation check}

As expected, there was a main effect of mood induction on mood, $F(1,124)=14.94, p<.001$. Participants in the positive condition were more positive $(M=5.35$, $\mathrm{SD}=.81)$ than participants in the neutral condition $(M=4.60, \mathrm{SD}=.93)$. In addition, the attractive comparison target was rated more attractive than the $(M=5.58$, $\mathrm{SD}=.78)$ than the unattractive target $(M=2.99$, $\mathrm{SD}=.89), F(1,110)=259.08, p<.001$. No other effects on ratings of attractiveness or mood were significant, $F \mathrm{~s}<1$.

\section{Self-evaluations}

Analysis revealed the expected three-way interaction effect of load, mood, and direction of comparison on attractiveness, $F(1,110)=11.23, p=001$. The effects of load, $F(1$, $110)=8.00, p=.006 ; \operatorname{mood} F(1,110)=9.83, p=.002$; direction, $F(1,110)=135.31, p<.001$; load and mood, $F(1,110)=10.97, p=.001 ;$ load and direction, $F(1$, $110)=10.44, p=.002$ and $\operatorname{mood}$ and direction, $F(1$, $110)=4.14, p=.04$, were also significant.

For ease of interpreting the three-way interaction, the pattern of means is presented in Fig. 3, and the cognitive load and no load conditions are analyzed separately, with comparisons to the control condition. ${ }^{5}$

No load conditions All participants in the study believed they shared a birthday with the comparison targets; therefore, the positive mood conditions were expected to replicate the positive mood and shared-birthday conditions in Experiment 1.

The expected asymmetry among positive mood participants was found. Higher levels of self-evaluations relative to the control condition $(M=4.20, \mathrm{SD}=.21)$ were found, regardless of whether participants upwardly compared $(M=5.64, \quad \mathrm{SD}=.78 ; \quad F(1,124)=17.39, p<.001)$ or downwardly compared $(M=5.15, \quad \mathrm{SD}=.81)$, $F(1,124)=11.90, p=.001$.

\footnotetext{
5 Again, in consideration of concerns about experimentwise alpha error, more conservative tests of the mean differences using the Tukey's HSD were conducted. These analyses revealed a similar pattern of significant differences from the control condition, with the only difference being that the high load, neutral mood induction, downward comparison condition differed from the control at the $p=.13$ level as opposed to the $p<.05$ level.
}

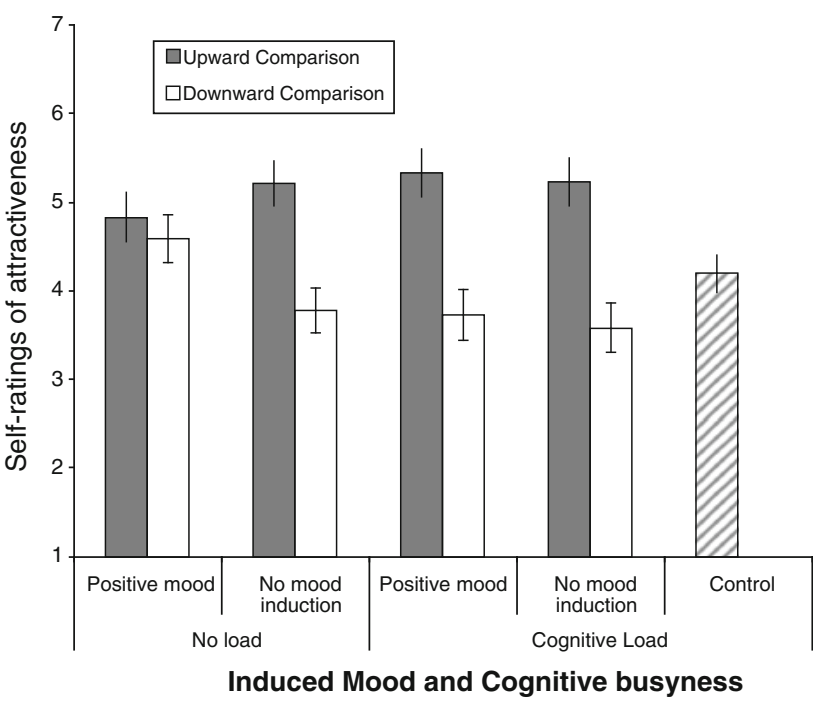

Fig. 3 Asymmetric responses to comparisons in positive mood are moderated by cognitive load; responses without mood induction are not. From Experiment 4

The neutral mood conditions replicated previous findings of symmetric assimilation (Brown et al. 1992). Upward comparison led to more positive self-ratings $(M=5.47, \quad \mathrm{SD}=.51)$ than the control condition, $F(1,124)=17.89, p<.001$, and downward comparison led to less positive self-ratings than the control condition $(M=3.24, \mathrm{SD}=1.09), F(1,124)=10.37, p=.002$.

Thus, positive mood led to asymmetric and self-serving responses, whereas neutral mood led to symmetric responses.

Cognitive load conditions Cognitive load was expected to disrupt the self-serving processing of participants in a positive mood, leading them to respond in the same manner as those in the neutral mood conditions.

As expected, cognitive load disrupted the self-serving responding associated with a positive mood. Instead, participants in a positive mood assimilated to both upward and downward comparison targets. Compared to a control condition $(M=4.20, \mathrm{SD}=.21)$, upward comparison led to more positive self-ratings $(M=5.71, \mathrm{SD}=.83)$, $F(1,124)=23.14, p<.001$, and downward comparison led to less positive self-ratings $(M=3.09, \mathrm{SD}=.94)$, $t(124)=-3.30, p=.001$. This pattern was identical to the neutral mood condition, in which upward comparison led to more positive self-ratings $(M=3.33, \mathrm{SD}=1.05)$, $F(1,124)=18.83, p<.001$, and downward comparison led to less positive self-ratings $(M=5.53, \mathrm{SD}=.87)$, $F(1,124)=7.84, \quad p=.006$, relative to the control condition.

Experiment 4 demonstrated that self-serving responding associated with positive mood is resource-dependent. 
When people in a positive mood were not under additional cognitive load, they adjusted their interpretations of the meaning of a shared birthday and responded to comparison targets in a self-serving ways, replicating Experiments 1 and 2. However, when cognitive resources were reduced, it was more difficult to adjust the interpretation of the meaning of a shared a birthday and, consequently, participants did not respond to comparisons in self-serving ways. Instead, their responses were like those of neutral mood participants: They assimilated to both upward and downward comparison targets. Thus, when cognitive resources were lacking, the benefits of a positive mood were muted.

\section{General discussion}

In four studies, positive mood led to self-serving interpretations of ambiguous similarity information. When participants were given ambiguous similarity information, they categorized targets in self-serving ways. If the comparison person was superior, they used the information to make the target distinctively similar, basked in that person's glory, and boosted their self-evaluations. If the comparison person was inferior, they used the information to make the target either irrelevant or only generally similar, leading to no response to the comparison target or contrast of their self-evaluations with the inferior target. Overall, this pattern of self-serving responding was not found when the preference information was unambiguous or when participants were under cognitive loaded.

These studies establish that mood can determine how people interpret and respond to comparison information. These studies also revealed boundary conditions to selfserving responding that provide insight into the underlying processes. First, self-serving responses only occurred when individuals were given ambiguous or flexible information about the relation between themselves and a comparison target. In Experiment 1, when individuals were not told that they shared a birthday with the comparison targets, they were unable to respond in a self-serving manner. Likewise, in Experiment 3, when similarity information was unambiguous, they did not respond in a self-serving manner. Thus, while positive mood might provide cognitive flexibility, positive mood does not blind individuals from seeing negative information. In the absence of ambiguous information, self-serving responses did not arise.

Second, the effect only occurred when individuals had adequate cognitive resources. That cognitive load derailed the self-serving responses suggests that the flexible processing of comparison targets in positive moods is an effortful process and may be a correction process (e.g., Gilbert et al. 1995; Stapel and Marx 2007). In Experiment 4 , in the absence of adequate cognitive resources, positive mood participants' responses to the comparison targets were similar to the responses to neutral mood participants. That is, they were unable to make corrections or engage in reinterpretation of ambiguous information in order to avoid assimilation to the downward comparison target. This supports our suggestion of a two-step process in which individuals in a positive mood initially respond to social comparison information in a consistent manner, and only engage in effortful reanalysis of ambiguous information in order to correct for the negative influence of social comparison.

In addition, this finding contradicts an alternative explanation that our effects are the result of positive moods inducing heuristic processing and negative moods inducing systematic processing. If heuristic processing were responsible for how participants responded to the comparisons, we would expect the same level of processing of both upward and downward comparisons, with symmetric contrast (or assimilation) to both types of targets. However, positive moods led to the use of a trivial similarity cue to indicate similarity in the upward comparison condition but not in the downward comparison conditions. In addition, if heuristic processing were responsible for the effects in the positive mood conditions, then inducing cognitive load should not have eliminated the self-serving pattern.

Third, we found asymmetry only under positive mood, not under negative mood. If self-serving responses were only defensive, we might have found that negative mood, an aversive state, would have led to an interpretation of information that allowed for recovery. Alternatively, if responses resulted from mood-congruent information search and processing, we would expect that negative mood should lead to asymmetrically negative responses. Instead, negative mood was associated with processing of information with greater neutrality, objectivity, and focus on the data at hand, regardless of the consequence for selfevaluations (for reviews see Bless 2001; Martin and Clore 2001).

Finally, the results of the present studies suggest that asymmetric responses are not a result of using mood as a resource (Trope et al. 2001). According to this model, a positive mood can be a treated as resource to be expended in the pursuit of accurate information. If this were true, in this case, we would have expected that positive mood would have been associated with contrastive responses to comparison targets, a response that is associated with information-seeking motives (Stapel and Koomen 2001a). Indeed, in other research, the experience of self-affirmation is related to just such symmetric responses to comparisons (Johnson and Stapel, in press).

In sum, these findings support the growing body of literature showing that positive moods can create an upward spiral (Fredrickson and Joiner 2002). Numerous cognitive 
processes are associated with positive mood and in responding to social comparison information; among these the ability to make fine distinctions between distinctive similarity and general similarity may be particularly helpful. Individuals in a positive mood were able to convert what could be considered negative feedback (one is less attractive than another person) into positive self-evaluations. Positive mood granted individuals the ability to see a shared-birthday or a shared-artistic preference as meaningful indicators of shared distinctiveness and similarity, allowing them to assimilate to upward comparison targets. Positive mood also granted individuals the ability to recognize a shared-birthday a meaningless indicator of similarity, and allowed them to contrast with downward comparison targets.

Future research might investigate how dispositional happiness might be related to the self-serving bias presented here. If positive emotions do create an upward spiral, consistently interpreting ambiguity in self-serving ways might one way in which dispostionally happy people are able to reap self-evaluative boosts from downward comparisons and are buffered from the self-depreciating effects of upward comparisons (Lyubomirsky and Ross 1997).

\section{Conclusions}

It has become increasingly clear that while social comparisons may be ubiquitous, the impact of social comparison information is highly dependent upon the contexts within which comparisons arise and the motivations of the individuals involved. The present studies add to the existing literature by highlighting how mood can influence how individuals treat similarity cues in their environment, such as social comparison information. In the presence of a positive mood, ambiguous social comparison information is interpreted in a self-serving manner, such that positive selfevaluations result. Thus, a good mood can beget not only a good mood, but also beget a more positive self-image.

Open Access This article is distributed under the terms of the Creative Commons Attribution Noncommercial License which permits any noncommercial use, distribution, and reproduction in any medium, provided the original author(s) and source are credited.

\section{References}

Avramova, Y. R., \& Stapel, D. A. (2008). Moods as spotlights: The influence of moods on accessibility effects. Journal of Personality and Social Psychology, 95, 542-554.

Bless, H. (2001). The consequences of mood on the processing of social information. In A. Tesser \& N. Schwarz (Eds.), Blackwell handbook of social psychology: Intraindividual processes (pp. 479-499). Malden, MA: Blackwell.

Brown, J. D., Novick, N. J., Lord, K. A., \& Richards, J. M. (1992). When Gulliver travels: Social context, psychological closeness, and self-appraisals. Journal of Personality and Social Psychology, 62, 717-727.

Caruso, E., Mead, N., \& Balcetis, E. (2009). Political partisanship influences perception of biracial candidates' skin tone. Proceedings of the National Academy of Sciences, 106, 20168-20173.

Cialdini, R. B., \& de Nicholas, M. E. (1989). Self-presentation by association. Journal of Personality and Social Psychology, 57(4), 626-631.

Clore, G. L., Gasper, K., Garvin, E., \& Forgas, J. P. (2001). Affect as information. In Handbook of affect and social cognition. (pp. 121-144). Mahwah, NJ, USA: Lawrence Erlbaum Associates Publishers.

Festinger, L. (1954). A theory of social comparison processes. Human Relations, 7, 117-140.

Finch, J. F., \& Cialdini, R. B. (1989). Another indirect tactic of (self-) image management: Boosting. Personality and Social Psychology Bulletin, 15(2), 222-232.

Förster, J., Liberman, N., \& Kuschel, S. (2008). The effect of global versus local processing styles on assimilation versus contrast in social judgment. Journal of Personality and Social Psychology, 94, 579-599.

Fredrickson, B. L. (2001). The role of positive emotions in positive psychology: The broaden-and-build theory of positive emotions. American Psychologist, 56(3), 218-226.

Fredrickson, B. L., \& Joiner, T. (2002). Positive emotions trigger upward spirals toward emotional well-being. Psychological Science, 13(2), 172-175.

Gasper, K. (2003). When necessity is the mother of invention: Mood and problem solving. Journal of Experimental Social Psychology, 39(3), 248-262.

Gasper, K., \& Clore, G. L. (2002). Attending to the big picture: Mood and global versus local processing of visual information. Psychological Science, 13(1), 34-40.

Gilbert, D. T., Giesler, R. B., \& Morris, K. A. (1995). When comparisons arise. Journal of Personality and Social Psychology, 69, 227-236.

Hirt, E. R., Devers, E. E., \& McCrea, S. M. (2008). I want to be creative: Exploring the role of hedonic contingency theory in the positive mood-cognitive flexibility link. Journal of Personality and Social Psychology, 94(2), 214-230.

Isbell, L. M. (2004). Not all happy people are lazy or stupid: Evidence of systematic processing in happy moods. Journal of Experimental Social Psychology, 40(3), 341-349.

Isen, A. M., Daubman, K. A., \& Nowicki, G. P. (1987). Positive affect facilitates creative problem solving. Journal of Personality and Social Psychology, 52(6), 1122-1131.

Jaccard, J. (1998). Interaction effects in factorial analysis of variance. Thousand Oaks, CA, USA: Sage.

Johnson, C. S., \& Stapel, D. A. (2007). When different is better: Performance following upward comparison. European Journal of Social Psychology, 37(2), 258-275.

Johnson, C. S., \& Stapel, D. A. (in press). Reflection vs. selfreflection: Sources of self-enhancement determine behavioral outcomes. Social Psychology.

Johnson, C. S., \& Stapel, D. A. Positive affect and affirmation are not the same. Unpublished manuscript, San Jose State University.

Kahneman, D., \& Tversky, A. (1973). On the psychology of prediction. Psychological Review, 80, 237-251. doi:10.1037/ h0034747.

Krauth-Gruber, S., \& Ric, F. (2000). Affect and stereotypic thinking: A test of the mood-and-general-knowledge model. Personality 
and Social Psychology Bulletin, 26, 1587-1597. doi: 10.1177/01461672002612012.

Lockwood, P., \& Kunda, Z. (1997). Superstars and me: Predicting the impact of role models on the self. Journal of Personality and Social Psychology, 73(1), 91-103.

Lyubomirsky, S., \& Ross, L. (1997). Hedonic consequences of social comparison: A contrast of happy and unhappy people. Journal of Personality and Social Psychology, 73(6), 1141-1157.

Martin, L. L., \& Clore, G. L. (2001). Theories of mood and cognition: A user's guidebook. Mahwah, NJ, USA: Lawrence Erlbaum Associates.

Miller, D. T., Turnbull, W., \& McFarland, C. (1988). Particularistic and universalistic evaluation in the social-comparison process. Journal of Personality and Social Psychology, 55(6), 908-917.

Mosak, H. H., \& Dreikurs, R. (1973). Adlerian psychotherapy. In R. Corsini (Ed.), Current psychotherapies (pp. 52-95). Hasca, IL: Peacock.

Murray, N., Sujan, H., Hirt, E. R., \& Sujan, M. (1990). The influence of mood on categorization - a cognitive flexibility interpretation. Journal of Personality and Social Psychology, 59(3), 411-425.

Mussweiler, T. (2003). Comparison processes in social judgment: Mechanisms and consequences. Psychological Review, 110(3), 472-489.

Schwarz, N. (1990). Feelings as information: Informational and motivational functions of affective states. New York, NY: Guilford Press.

Schwarz, N., \& Clore, G. L. (1983). Mood, misattribution, and judgments of well-being: Informative and directive functions of affective states. Journal of Personality and Social Psychology, 45(3), 513-523.

Schwinghammer, S. A., Stapel, D. A., \& Blanton, H. (2006). Different selves have different effects: Self-activation and defensive social comparison. Personality and Social Psychology Bulletin, 32(1), 27-39.

Stapel, D. A., \& Blanton, H. (2004). From seeing to being: Subliminal social comparisons affect implicit and explicit self-evaluations. Journal of Personality and Social Psychology, 87(4), 468-481.
Stapel, D. A., \& Johnson, C. S. (2007). When nothing compares to me: How defensive motivations and similarity shape social comparison effects. European Journal of Social Psychology, 37(5), 824-838.

Stapel, D. A., \& Koomen, W. (2000). Distinctness of others, mutability of selves: Their impact on self-evaluations. Journal of Personality and Social Psychology, 79(6), 1068-1087.

Stapel, D. A., \& Koomen, W. (2001a). I, we, and the effects of others on me: How self-construal level moderates social comparison effects. Journal of Personality and Social Psychology, 80, 766-781.

Stapel, D. A., \& Koomen, W. (2001b). The impact of interpretation versus comparison mindsets on knowledge accessibility effects. Journal of Experimental Social Psychology, 37(2), 134-149.

Stapel, D. A., \& Marx, D. M. (2007). Distinctiveness is key: How different types of self-other similarity moderate social comparison effects. Personality and Social Psychology Bulletin, 33(3), 439-448.

Storbeck, J., \& Clore, G. L. (2005). With sadness conies accuracy; with happiness, false memory: Mood and the false memory effect. Psychological Science, 16(10), 785-791.

Tamir, M., \& Robinson, M. D. (2007). The happy spotlight: Positive mood and selective attention to rewarding information. Personality and Social Psychology Bulletin, 33(8), 1124-1136.

Trope, Y., Ferguson, M., \& Raghunathan, R. (2001). Mood as a resource in processing self-relevant information. In J. P. Forgas (Ed.), Handbook of affect and social cognition (pp. 256-274). Mahwah, NJ, USA: Lawrence Erlbaum Associates.

Wills, T. A. (1981). Downward comparison principles in social psychology. Psychological Bulletin, 90(2), 245-271.

Wood, J. V. (1989). Theory and research concerning social comparison of personal attributes. Journal of Personality and Social Psychology, 106(3), 231-248. 